\title{
The elastic modulus of columnar-grain fresh-water ice
}

\author{
LORNE W. GOLD \\ Cold Regions Engineering Program, Institute for Mechanical Engineering, National Research Council of Canada, \\ Ottawa, Ontario, K1A OR6 Canada
}

\begin{abstract}
An analysis is presented of stress and strain measurements made during an investigation of the characteristics of cracks formed in columnar-grain, type S2, fresh-water ice, during uniaxial, compressive loading at the nominal strain rates of $10^{-3}, 10^{-4}$ and $10^{-5} \mathrm{~s}^{-1}$, and temperatures of $-5^{\circ},-10^{\circ},-20^{\circ}$ and $-30^{\circ} \mathrm{C}$. The analysis shows that for this range of strain rate and temperature, ice behaves as an anelastic solid. Results are given for the time, grain-size and temperature dependence of the elastic modulus in the plane perpendicular to the long direction of the grains. They are shown to be in reasonable agreement with results of an earlier study of the anelastic behaviour of the same type of ice. It is suggested that the grain-size and temperature dependence of the elastic moduli of ice for this range of strain rate and temperature may be due, in part, to the dependence of the relaxation time on these variables.
\end{abstract}

\section{INTRODUCTION}

An investigation was carried out at the Institute for Marine Dynamics, National Research Council of Canada, on crack formation in columnar-grain, type S2, fresh-water ice. The principal objective of the work was to obtain information on the characteristics of the cracks that are formed by a compressive stress applied perpendicular to the long direction of the grains. The investigation included mesurement of the stress and strain. This paper presents an analysis of these measurements.

The crystallographic $c$ axis of the grains of type S2 ice tends to lie in the plane perpendicular to the long direction of the grains and to have a random orientation in that plane. Because of this preferred orientation, its mechanical response to an applied load is transverse isotropic. If a load is applied perpendicular to the long direction of the grains, the first $1 \%$ of deformation is practically two-dimensional, i.e. the strain in the long direction of the grains is much less than in the plane perpendicular to that direction. The information in this paper is for the time-dependent elastic modulus in the plane perpendicular to the long direction of the grains which, because of the symmetry, is independent of direction in that plane.

Traetteberg and others (1975) carried out a study of the strain rate and temperature dependence of the elastic modulus of type $\mathrm{S} 2$ ice. Their measurements were made in the strain-rate range of $10^{-8}$ to $5 \times 10^{-3} \mathrm{~s}^{-1}$, and in the temperature range of $-10^{\circ}$ to $-40^{\circ} \mathrm{C}$. The maximum stress applied was $0.5 \mathrm{MPa}$. Gold and Traetteberg (1975) showed that the time dependence of the elastic moduli found by Traetteberg and others could be described by anelastic theory. This theory is the basis for the analysis of the present work. Following a review of some of the results of the earlier work, results from the present investigation are compared to them. Information obtained by Traetteberg on the anelastic behaviour of granular ice is given also, but only for purposes of comparison with that for the columnar-grain ice.

Much attention is given to the development of rheological models for ice. There are still differences of opinion over the form of the expressions for the delayed elastic and viscous components of the strain. One reason for this, for the delayed elastic component, is that we do not have a fully satisfactory understanding of its dependence on ice type, time, temperature and grainsize. It is shown in this paper that the theory of the anelastic behaviour of solids, which considers only the elastic and delayed elastic components of the total strain, provides considerable insight concerning this dependence.

\section{PREPARATION OF THE ICE}

The ice was made from deionized and de-aerated water in an open-top, insulated fibreglass tank $55 \times 35 \times 25 \mathrm{~cm}$. The water was cooled in a cold room until ice began to form on the surface. This ice was removed quickly, and the surface covered with ice particles of a given range in size obtained by crushing and sieving large single crystals. As the water froze below the crushed ice layer, type S2 ice developed within about $3 \mathrm{~cm}$ of it. This ice was usually free of visible bubbles to a thickness of $15 \mathrm{~cm}$. The average cross-sectional area of the grains near the surface was determined by the size of the seed particles, and increased gradually in the direction of freezing.

Rectangular specimens $30 \times 90 \times 200 \mathrm{~mm}$ were used for the tests. The long direction of the grains was perpendicular to the $90 \times 200 \mathrm{~mm}$ face. After the seed and transition layers were removed, it was usually 
possible to make eight specimens from each block of ice made in the tank. The specimens were formed in a milling machine so that the faces and sides were parallel to within $0.1 \mathrm{~mm}$ and the ends to better than $0.02 \mathrm{~mm}$. After machining, each specimen was sealed in a plastic bag and stored in an airtight box. It was conditioned at the test temperature for at least $24 \mathrm{~h}$ prior to testing.

Average grain-size was determined in the following manner from photographs of thin sections cut from the central $90 \times 90 \mathrm{~mm}$ of the two faces of each specimen after it was tested. The average grain area for each face was obtained by counting the number of grains in a given area, and was used to calculate the diameter of the circle of equal area. The average grain-size was taken to be the mean of the diameters calculated for the two faces.

\section{DESCRIPTION OF THE EXPERIMENTS}

Tests were carried out at constant rate of cross-head movement in a closed-loop testing machine (MTS $311.31)$. Compressive loads were applied to the $30 \times$ $90 \mathrm{~mm}$ ends of the specimens. They were limited to between 3 and $5 \mathrm{MPa}$, depending on the nominal strain rate and temperature. Strain was measured with LVDT extensometers with a gauge length of $60 \mathrm{~mm}$, mounted on each side of the specimen. Tests were carried out at the nominal strain rates of $10^{-3}, 10^{-4}$ and $10^{-5} \mathrm{~s}^{-1}$ at the temperature of $-10^{\circ} \mathrm{C}$. The corresponding measured strain rates were about $7.6 \times 10^{-4}, 7.6 \times 10^{-5}$ and $7.8 \times 10^{-6} \mathrm{~s}^{-1}$. They were found to be almost constant over the strain range of $1.5 \times 10^{-4}$ to the maximum amount of between $4.5 \times 10^{-4}$ and $7 \times 10^{-4}$. Tests were carried out also at the nominal rate of $10^{-3} \mathrm{~s}^{-1}$ at temperatures of $-5^{\circ},-20^{\circ}$ and $-30^{\circ} \mathrm{C}$. The output from the two extensometers, the load cell and the piston displacement transducer were sampled at the rate of 30 to $200 \mathrm{~Hz}$, depending on the nominal strain rate and temperature, and stored in a computer. The strain used in the analysis was the mean of the values measured by the two extensometers.

Loads were applied through steel platens. The lower one was fixed and mounted on a load-cell. The upper platen had a non-locking ball and cone joint to ensure full contact with the ends of the specimen. A very small preload, less than $0.1 \mathrm{MPa}$ and small enough to allow the position of the specimen to be adjusted to the centre of the lower platen, was applied for about $5 \mathrm{~min}$ before the beginning of each test.

Twenty-one tests were conducted at $-10^{\circ} \mathrm{C}$ and nominal strain rate of $10^{-3} \mathrm{~s}^{-1}$ and 14 at each of the two other nominal rates. The average grain-size for these tests was in the range of 2.9 to $6.2 \mathrm{~mm}$.

\section{RESULTS}

Plots of stress vs time, stress vs strain, strain vs time for each extensometer, and the mean of the two strain readings, were prepared from the stored data. It was found for some tests that the strain calculated from one extensometer was displaced by a small amount relative to the other, indicating that the specimen for these tests may
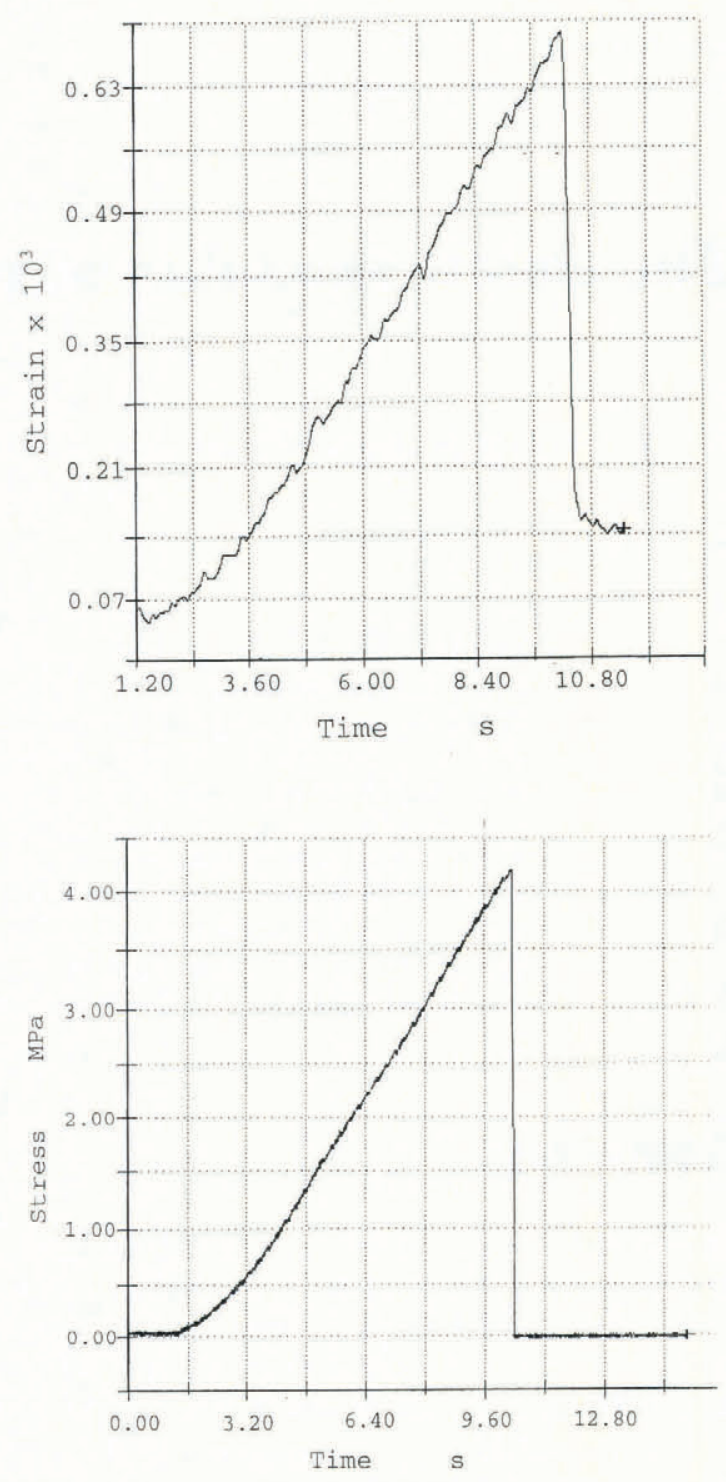

b

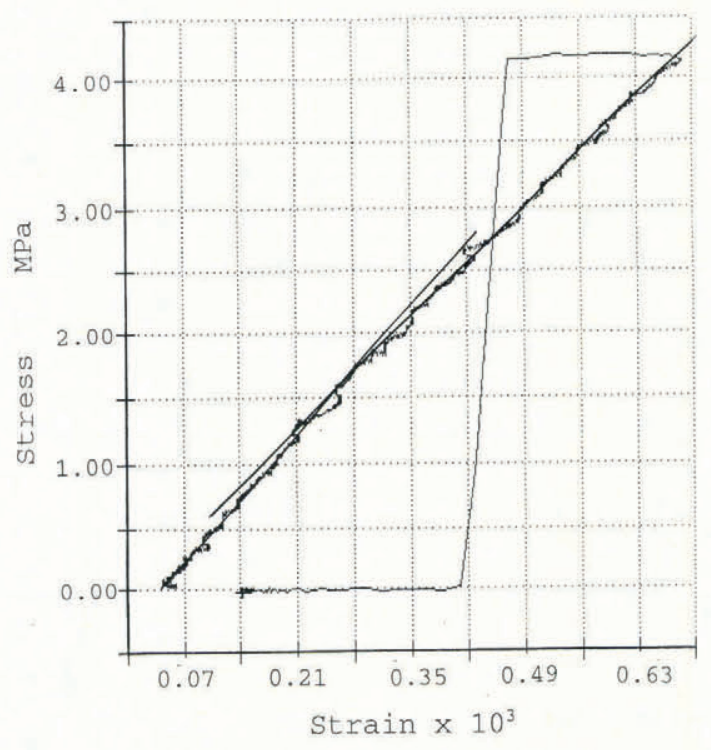

Fig. 1. An example of the time dependence of the stress and the average strain, and of the dependence of the strain on stress. The initial tangent modulus, $E_{1}$, and the subsequent modulus, $E_{2}$, are equal to the slope of the two lines drawn through the corresponding, effectively linear, sections of the stress vs strain curve. Nominal strain rate, $10^{-4} \mathrm{~s}^{-1}$; temperature, $-10^{\circ} \mathrm{C}$. 
not have been in the centre of the platen. The difference in the two strain readings was usually less than $5 \times 10^{-5}$. For practically all the tests except those at the lowest nominal strain rate, however, the strain vs time curves were parallel after the small displacement. For some of the tests at the lowest rate the curves were not parallel, indicating a difference in strain rate between the two sides of the specimen.

Both the stress and the strain rates increased with time at the beginning of each test, then tended to constant values. They were effectively constant for most of the test at the two highest nominal strain rates, but not for the lowest one. The stress vs strain curves had corresponding, effectively linear sections, indicating a relatively small change of the anelastic modulus with time. An example of the dependence of the strain on stress and of the time dependence of the stress and average strain, is shown in Figure 1 for a test at the nominal rate of $10^{-4} \mathrm{~s}^{-1}$.

It was found necessary to smooth the stress and strain data because of small, relatively high frequency fluctuations in the records. The effect of these fluctuations was less for the stress vs strain plots, but it did require that moduli be determined for relatively large ranges of stress and strain, as can be appreciated from Figure 1. In effect, what was obtained for each specimen was the tangent value for the time associated with the midpoint of the selected ranges of strain.

The modulus, $E_{1}$, given by the slope of the tangent to the initial part of the stress-strain curve, was determined for each test. A modulus, $E_{2}$, was calculated from the effectively linear part of the stress-strain curve that corresponded to the time range for which both the stress and the strain rates could be assumed constant. These two moduli described the major part of the stress-strain behaviour for the higher nominal strain rates, as can be seen from Figure 1. For the lowest nominal rate, a third modulus was determined to characterize the stress-strain behaviour near the maximum imposed strain.

Figure 2 presents the grain-size dependence and associated regression equation found for $E_{1}$ at the nominal rate of $10^{-3} \mathrm{~s}^{-1}$ and temperature of $-10^{\circ} \mathrm{C}$. The regression equations for the three nominal rates, with the

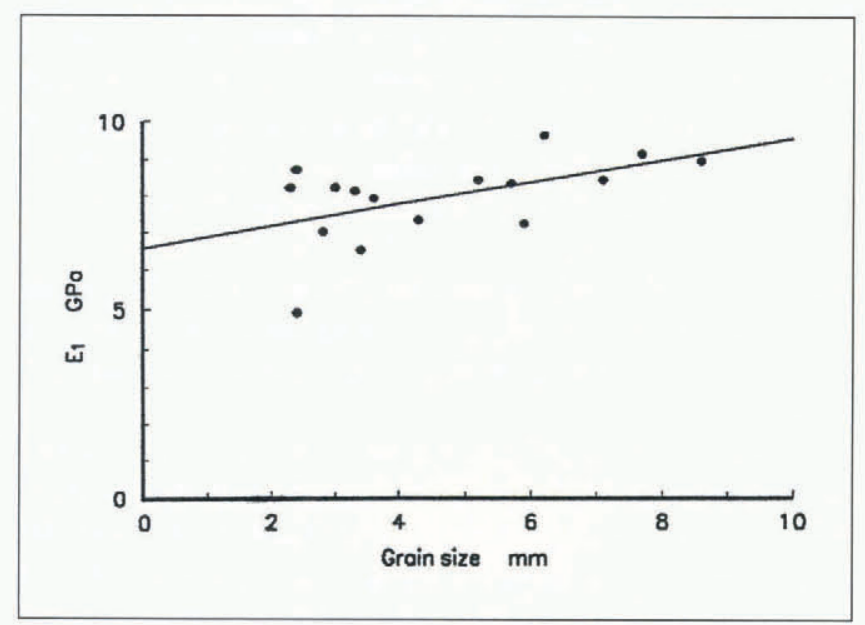

Fig. 2. Grain-size dependence of the initial elastic modulus, $E_{1}$, and plot of the associated regression equation. Nominal strain rate, $10^{-3} \mathrm{~s}^{-1}$; temperature, $-10^{\circ} \mathrm{C}$.
Table 1. Regression equations giving the dependence of the elastic moduli, $E_{1}$ and $E_{2}$, on average grain-size, $d$, for columnar-grain ice and three nominal rates of strain, with their standard deviations, $S_{E d}$, and coefficients of correlation, $r$; temperature, $-10^{\circ} \mathrm{C}$

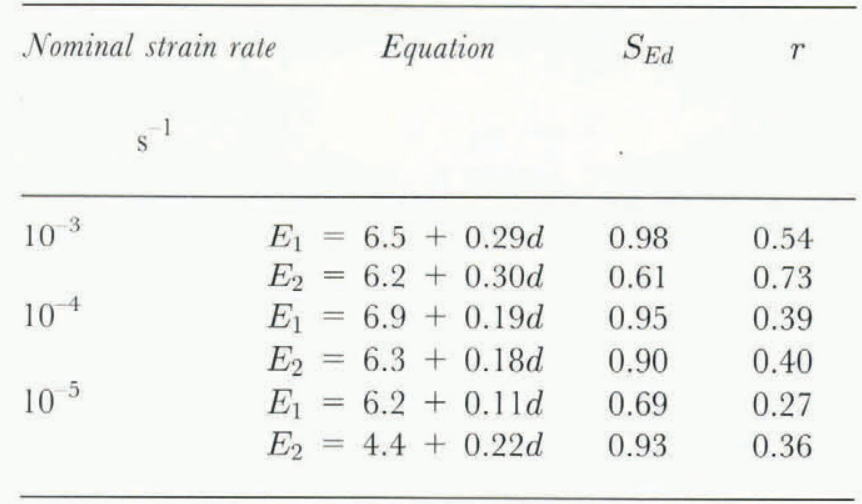

associated standard deviations and correlation coefficients, are given in Table 1 . It can be seen that there is a dependence of the modulus on grain-size for all three rates, but it becomes weaker with decreasing strain rate.

The grain-size dependence for $E_{2}$, and associated regression equation, is shown in Figure 3 for the nominal rate of $10^{-3} \mathrm{~s}^{-1}$; the regression equations and associated standard deviations and correlation coefficients are presented for the three rates in Table 1. It can be seen

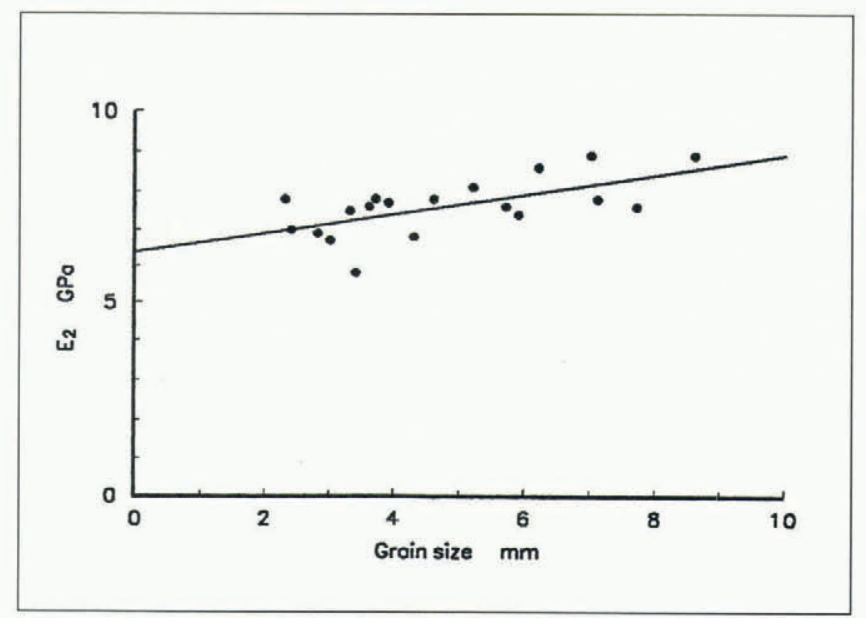

Fig. 3. Grain-size dependence of the elastic modulus, $E_{2}$, and plot of the associated regression equation. Nominal strain rate $10^{-3} \mathrm{~s}^{-1}$; temperature, $-10^{\circ} \mathrm{C}$.

that $E_{2}$ has about the same dependence on grain size as $E_{1}$. The dependence of the average value of $E_{1}$ and $E_{2}$ on temperature, at the nominal rate of $10^{-3} \mathrm{~s}^{-1}$ and grain-size 2.9 to $6.2 \mathrm{~mm}$, is shown in Figure 4 . Shown also is the temperature dependence found by Traetteberg and others (1975) and Gold (1958) from measurements made at about the same time after the initiation of a constant rate of stressing.

\section{ANALYSIS}

Gold and Traetteberg (1975) assumed that the initial response of ice was anelastic and could be described by 


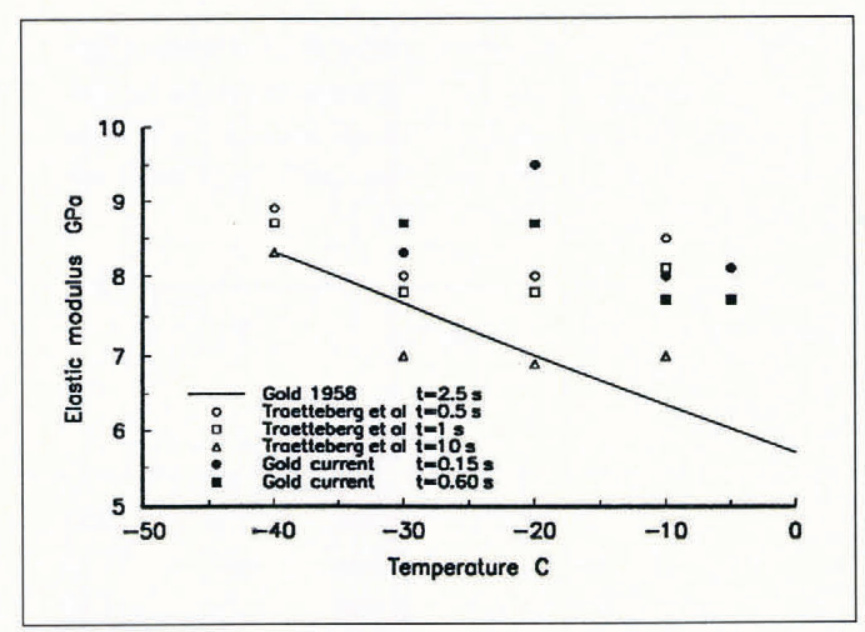

Fig. 4. Temperature dependence of the average elastic moduli $E_{1}$ (solid circle) and $E_{2}$ (solid square), measured by Traetteberg and others (1975) and Gold (1958). The time associated with each of the data points is shown in the legend; the average grain-size of the specimens was in the range of 3 to $6 \mathrm{~mm}$.

the following equation (Nowick and Berry, 1972):

$$
\sigma+\tau_{\varepsilon} \dot{\varepsilon}=M_{\mathrm{R}}\left(\varepsilon+\tau_{\sigma} \dot{\varepsilon}\right)
$$

where $\varepsilon$ is the strain, $\sigma$ is the stress, $\tau_{\varepsilon}$ the relaxation time for constant strain, $\tau_{\sigma}$ the relaxation time for constant stress, $M_{\mathrm{R}}$ the relaxed modulus, and the dot indicates differentiation with respect to time. They determined values of $\tau_{\sigma}$ from the strain relaxation after the load was removed completely. The dependence of $\tau_{\sigma}$ on the time of duration of the test, obtained for both columnar-grain, type $\mathrm{S} 2$ ice, and granular ice, is shown in Figure 5. It was found that the relaxation time was not constant but increased with time raised to the power of about $2 / 3$. A line with this slope has been drawn through the data in Figure 5. With this dependence on time, Equation (1) becomes:

$$
\sigma+\alpha_{\varepsilon} t^{2 / 3} \dot{\sigma}=M_{\mathrm{R}}\left(\varepsilon+\alpha_{\sigma} t^{2 / 3} \dot{\varepsilon}\right)
$$

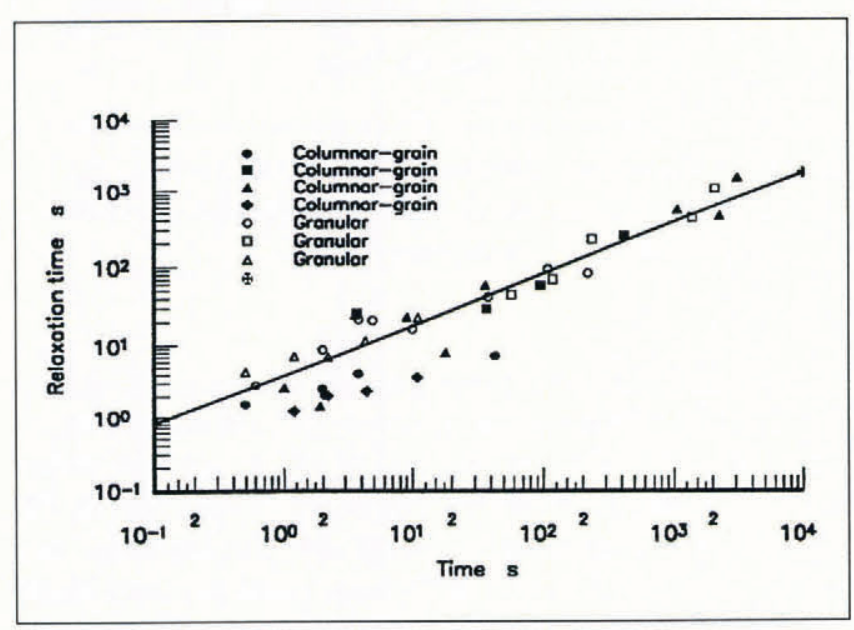

Fig. 5. Time dependence of the relaxation time, determined from the measurements of Traetteberg (Traetteberg and others, 1975). The same symbol is for a series of consecutive cycles of load applied at different, almost constant, rate of increase of the stress. Temperature, $-10^{\circ} \mathrm{C}$. where $\alpha_{\varepsilon} t^{2 / 3}=\tau_{\varepsilon}$ and $\alpha_{\sigma} t^{2 / 3}=\tau_{\sigma}$.

It was observed that the rate of change of the stress was almost constant during most of the application and removal of the load for the tests from which the relaxation times, shown in Figure 5, were determined. Gold and Traetteberg obtained the following solution for Equation (2) for the period during which the load was applied, using Laplace transforms and assuming that the rate of increase of the stress was constant:

$$
\begin{aligned}
\varepsilon(t)= & \frac{\sigma_{0}}{M_{\mathrm{R}}}\left(t+\left(\alpha_{\sigma}-\alpha_{\varepsilon}\right)\right. \\
& {\left.\left[\frac{2}{3} \alpha_{\sigma} t^{1 / 3}-t^{2 / 3}+\frac{2}{9} \alpha_{\sigma}^{2}\left(\exp \left(-\frac{3 t^{1 / 3}}{\alpha_{\sigma}}\right)-1\right)\right]\right)(3) }
\end{aligned}
$$

where $\sigma_{0}$ is the constant rate of increase of the stress.

It can be appreciated from Equation (3) that it is more convenient to use the compliance than the anelastic modulus for strain calculations when the stress is the controlled variable. The compliance at time $t$ is given by $S(t)=\dot{\varepsilon}(t) / \sigma_{0}$. Differentiating Equation (3) with respect to time and rearranging gives:

$$
\begin{aligned}
& M_{\mathrm{R}} S(t)- 1=\frac{2}{3}\left(\alpha_{\sigma}-\alpha_{\varepsilon}\right) \\
& \cdot\left(\frac{\alpha_{\sigma} t^{-2 / 3}}{3}\left[1-\exp \left(\frac{-3 t^{1 / 3}}{\alpha_{\sigma}}\right)\right]-t^{-1 / 3}\right) .
\end{aligned}
$$

If the relaxation in the compliance is spread over several decades of time, however, as it is in the present case, $E(t)$ can be assumed equal to $1 / S(t)$ (see Nowick and Berry, 1972 , p. 35). Equation (4) shows that the compliance, and therefore the anelastic modulus, does not depend on the strain or stress rates for the condition analyzed, but on the duration of application of the constant rate of increase of the stress.

The time-dependent moduli, calculated from measurements made by Traetteberg and others (1975) for a specimen of columnar-grain, type S2 ice, are presented in Figure 6 . The columnar-grain specimen was subjected to over 23 load cycles, each at one of 13 crosshead rates. These were applied during three separate days of testing. Results obtained for a granular ice specimen subjected to 16 load cycles during two days of testing are shown as well. The specimens were annealed at least one week between sets of load cycles. The time associated with each value of the modulus was taken to be the logarithmic mean of the period for which the dependence of strain on stress was effectively linear. The results presented in Figure 6 differ a little from those in figure 3 of Gold and Traetteberg (1975) because of additional data for the columnar-grain ice and corrections for density for the granular ice.

Using the results from the measurements of Traetteberg presented in Figure 5, it was assumed that $\tau_{\sigma}=$ $3.8 t^{2 / 3} \mathrm{~s}$. Reasonable values for $E(t)$ for $t=0.1$ and $10 \mathrm{~s}$ were selected from Figure 6 for both types of ice and used to determine the constants $M_{\mathrm{R}}$ and $\alpha_{\varepsilon}$. Their values, which are a little different from those given by Gold and Traetteberg, are presented in Table 2 along with that of the unrelaxed modulus, $M_{\mathrm{U}}$, given by $M_{\mathrm{U}}=M_{\mathrm{R}} \tau_{\sigma} / \tau_{\varepsilon}$. $E(t)$, calculated from Equation (4), is plotted in the figure 


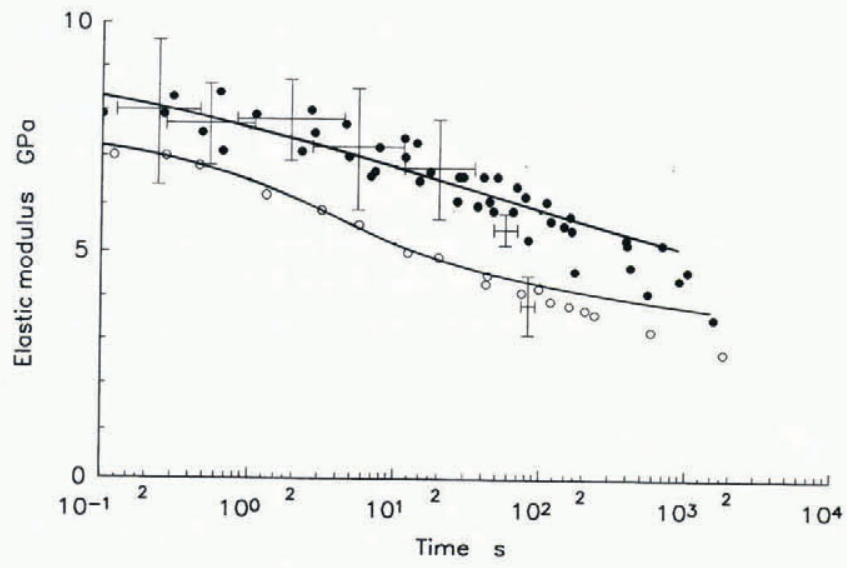

Fig. 6. Time dependence of the elastic moduli. The solid and open circles are values for columnnar-grain and granular ice, respectively, obtained from a re-analysis of the measurements made by Traetteberg (Traetteberg and others, 1975). The data points with the error bars are from the current work. For the first two on the left, the nominal strain rate was $10^{-3} \mathrm{~s}^{-1}$; for the third and fourth, $10^{-4} \mathrm{~s}^{-1}$; and for the fifth and sixth, $10^{-5} \mathrm{~s}^{-1}$. The seventh on the extreme right is the third modulus determined at the nominal rate of $10^{-5} \mathrm{~s}^{-1}$. Grain-size, $5 \mp 1 \mathrm{~mm}$; temperature, $-10^{\circ} \mathrm{C}$.

using these values for the constants. It can be seen that the equation is in good agreement with the earlier observations over the period of 0.1 to about $30 \mathrm{~s}$.

The columnar-grain ice used by Traetteberg had an average grain size of about $5 \mathrm{~mm}$. Values of $E_{1}$ and $E_{2}$ for an average grain-size of $5 \mathrm{~mm}$ were determined for the present work from the regression equations presented in Table 1. These are plotted in Figure 6. The bars associated with each point give the range in time and values of the elastic moduli observed for specimens of average grain-size in the range of $5 \pm 1 \mathrm{~mm}$. The data point on the extreme right is the average value of the third modulus determined in the present investigation at the nominal strain rate of $10^{-5} \mathrm{~s}^{-1}$ for specimens in the same range of average grain size. It can be seen that these average values agree well with the results of the earlier work over the range of 0.1 to about $30 \mathrm{~s}$. Thereafter, the values begin to deviate from the theoretical curve, but more rapidly than found by Gold and Traetteberg. This is

Table 2. The anelastic constants for columnar-grain and granular ice determined from the work of Traetteberg and others (1975) for constant rate of stress conditions; grainsize, about $5 \mathrm{~mm}$; temperature $-10^{\circ} \mathrm{C}$

\begin{tabular}{|c|c|c|c|c|}
\hline \multirow{3}{*}{ Type of ice } & \multicolumn{4}{|c|}{ Anelastic constants } \\
\hline & $M_{\mathrm{R}}$ & $M_{\mathrm{U}}$ & $\alpha_{\sigma}$ & $\alpha_{\varepsilon}$ \\
\hline & Gpa & $\mathrm{GPa}$ & $s^{1 / 3}$ & $s^{1 / 3}$ \\
\hline Columnar-grain & 4.8 & 9.1 & 3.8 & 2.0 \\
\hline Granular & 3.2 & 8.6 & 3.8 & 1.4 \\
\hline
\end{tabular}

because the viscous component of the total strain in the present study would be larger, due to the larger maximum stress.

\section{DISCUSSION}

The exponential term in Equation (3) has the same form as that given by Sinha (1979) for the delayed elastic component of his rheological equation. He obtained a value of 0.34 for the exponent for the time in the exponential term by fitting his equation to data from creep tests. In the present analysis, an exponent of $1 / 3$ occurs because of the time dependence of the relaxation time.

A direct comparison of Sinha's expression for delayed elasticity and Equation (3) requires further analysis because of differences in the stress conditions (Equation (3) is for a constant rate of increase of stress; Sinha's equation is for a constant stress). Sinha (1983), however, used a finite-difference method to show that his rheological equation agreed satisfactorily with the results of tests carried out at a constant rate of crosshead displacement on columnar-grain ice. In the limit, his summation for the delayed elastic term transforms to the Laplacian form. As the test conditions closely approximated those of constant load rate, it is reasonable to assume that his calculations simulated the response described by Equation (4).

The relaxation of the elastic moduli determined in this study takes place over the time range beginning about $10^{-1} \mathrm{~s}$ and extending beyond $10^{3} \mathrm{~s}$. Kê (1947) has shown that, for metals, the relaxation of the elastic moduli that occurs over this general range of time, is due to viscous processes on and near grain boundaries. Internal friction measurements made by Kuroiwa (1964) show that this is the case also for ice. The present results are consistent with those of Kuroiwa.

The temperature dependence found from the present studies is similar to that reported earlier by Traetteberg and others (1975) and Gold (1958), but there is considerable scatter. Measurements of anelastic behaviour have shown that, in many cases, the relaxation time has an Arrhenius relation with temperature (see Nowick and Berry, p. 57). This suggests, for ice, that the temperature dependence of the moduli during anelastic relaxation may be due to the temperature dependence of $\alpha_{\sigma}$ and $\alpha_{\varepsilon}$. This was demonstrated by Sinha (1978), where he determined the activation energy from the temperature dependence of the "shift function", which is the factor associated with the time in his delayed elastic equation, in the same manner as $3 \alpha_{\sigma}{ }^{-1}$ in the exponential term of Equation (3). Because the expression for the anelastic modulus for the constant rate of stressing condition is not simple, it may be better to determine the temperature dependence using the constant stress condition, as was done by Sinha, or by measuring the temperature dependence of the relaxation time. The latter is done most easily by measuring the temperature dependence of the internal friction at low frequency (less than $20 \mathrm{~Hz}$ ) or at small rates of stressing.

The work of Kê (1947) indicates that the relaxation time is directly proportional to grain-size. Nowick and 
Berry (1972) summarized evidence to show that the dependence may not be that simple. Sinha (1979) assumed that the shift function does not depend on the grain-size. Kuroiwa (1964) shows, on the other hand, that the relaxation depends not only on the grain-size, but on the impurity content of the ice as well. Careful measurements should be made to determine if the relaxation time for ice depends on grain-size and, if it does, to what extent does it account for the observed dependence for the anelastic modulus.

It can be seen in Figure 5 that the time dependence of the relaxation time is almost the same for each specimen during a set of load cycles. The position of each set of data points, however, changes from one set to another and with specimen. The values for granular ice tend to be larger than for columnar-grain ice, but no correction had been made for density. In addition, the study from which the relaxation times were determined indicated that the time dependence presented in this paper became fully established only after about five seconds into the relaxation process. This may be because the load conditions did not conform fully with those required for the method used to calculate the relaxation times, i.e. the load was removed at a constant rate and not abruptly. This data set indicated also that there is a significant memory effect associated with load changes. There was evidence, as well, that the behaviour after repeated load cycles was different from that for the first cycle. Clearly, more information is needed on the nature and effects of the relaxation processes that occur in ice during the period immediately following a change in stress or stress rate.

Cracks began to form in the specimens when the stress exceeded about $2 \mathrm{MPa}$, depending on strain rate, grainsize and temperature (Gold and others, 1992). For many specimens, the crack density was quite high when the loads were removed. For the nominal rates of $10^{-3}$ and $10^{-4} \mathrm{~s}^{-1}$, the maximum stress was about $75 \%$ of the brittle failure stress. The stress and strain measurements indicated that this cracking activity did not have a noticeable effect on the anelastic response of the ice. Although the stress levels were about ten times larger than those applied by Traetteberg and others, the elastic moduli determined from the two studies were in good agreement. These observations indicate that at high rates of strain, ice may behave as an anelastic solid right to failure,

A relatively abrupt change in the stress vs strain curve was observed for many of the tests at a stress of about $1 \mathrm{MPa}$. It could not be established if this change was due to deformation processes or, for example, to the load not being applied at the exact centre of the specimen. If it is real, however, it does not appear to be large enough to affect significantly the time dependence of the moduli shown in Figure 6.

Figure 6 shows that the viscous creep component of the strain becomes important in constant-stress-rate uniaxial deformation at temperatures lower than about $-5^{\circ} \mathrm{C}$, only when the time of application of the stress is greater than about $30 \mathrm{~s}$. There is a wide range of engineering problems involving ice for which the duration of the load is less than this time. The present study supports the position that only the elastic and delayed elastic components of the strain need be used for their analysis. This simplifies calculations of the timedependent response of ice to relatively rapidly applied loads, such as during impact with ships or fixed structures, or the deflection of ice covers under a moving load. A study that would be of interest for the brittle to ductile transition would be the dependence of the strain ratio on time alone, rather than on strain rate or stress rate.

\section{CONGLUSIONS}

Ice can be considered as an anelastic solid for the first $30 \mathrm{~s}$ of deformation under a uniaxial load applied at a constant rate of stress at temperatures equal to or lower than $-5^{\circ} \mathrm{C}$. Published information indicates that the associated relaxation processes occur on or in the vicinity of grain boundaries. Relaxation of the anelastic moduli begins less than $10^{-1} \mathrm{~s}$ and continues to $10^{3} \mathrm{~s}$, at least, after the application of the stress. The temperature and grainsize dependence of the moduli in the time range of about 0.1 to $30 \mathrm{~s}$ after the application of the load, may be due, at least in part, to the dependence of the relaxation time on these variables.

\section{ACKNOWLEDGEMENTS}

The author expresses his appreciation to the Institute for Marine Dynamics of the National Research Council of Canada and, in particular, to Dr Stephen Jones for the opportunity to carry out this research. He wishes to thank, as well, Trent Slade for his valuable assistance with preparing the specimens and carrying out the tests, and Dr Nirmal Sinha for his helpful comments.

\section{REFERENCES}

Gold, L. W. 1958. Some observations on the dependence of strain on stress for ice. Can. J. Phys., 36(10), 1265-1275.

Gold, L. W. and A. Traetteberg. 1975. Young's modulus of ice and ice engineering problems. In Weaver, D.S., ed. Proceedings. Second Symposium. Applications of Solid Mechanics, June 17 and 18, 1974. Vol. 1 . Hamilton, Ontario, McMaster University. Faculty of Engineering, $1-16$.

Gold, L. W., S. J. Jones and T. D. Slade. 1992. A comparison of crack initiation conditions for columnar-grain and granular ice. In Proceedings. Eleventh International Symposium on Ice Problems, 15-19 fune, Banff, Alberta. Vol. 1. International Association of Hydraulic Research, 200-209.

Kê, T. 1947. Experimental evidence of the viscous behavior of grain boundaries in metals. Phys. Rev., 71(8), 533-546.

Kuriowa, D. 1964. Internal friction of ice. Contrib. Inst. Low Temp. Sci., Ser. A 18.

Nowick, A. S. and B. S. Berry. 1972. Anelastic relaxation in crystalline solids. New York, Academic Press.

Sinha, N. K. 1978. Rheology of columnar-grained ice. Exp. Mech. $18(12), 464470$.

Sinha, N. K. 1979. Grain boundary sliding in polycrystalline materials Philos. Mag., Ser. A, $\mathbf{4 0}(6), 825-842$.

Sinha, N. K. 1983. Creep model of ice for monotonically increasing stress. Cold Reg. Sci. Technol., 8(1), 25-33.

Traetteberg, A., L. W. Gold and R. Frederking. 1975. The strain rate and temperature dependence of Young's modulus of ice. In Frankenstein, G. E., ed. Proceedings. Third International Symposium on Ice Problems, 1821 August 1975, Hanover, New Hampshire. International Association of Hydraulic Research, 479486.

The accuracy of references in the text and in this list is the responsibility of the author, to whom queries should be addressed. 